\title{
Experimental Study of Combined Effects of Gamma and Laser Radiations on the Properties of Silicate Glass Materials
}

\author{
R. T. Khaydarov ${ }^{1, *}$, H. B. Beisinbaeva ${ }^{1}$, R. R. Khaydarov ${ }^{1}$, F. R. Tojinazarov ${ }^{1}$, G. R. Berdiyorov ${ }^{2}$, \\ J. T. Berdiyorov ${ }^{3}$, Rahmatullaev I. ${ }^{1}$ \\ ${ }^{1}$ Institute of Applied Physics, National University of Uzbekistan, Uzbekistan \\ ${ }^{2}$ Qatar Environment and Energy Research Institute, Qatar Foundation, Qatar \\ ${ }^{3}$ Tashkent Polytechnical College, Uzbekistan
}

Copyright $(\mathcal{C} 2015$ by authors, all rights reserved. Authors agree that this article remains permanently open access under the terms of the Creative Commons Attribution License 4.0 International License

\begin{abstract}
Effect of ${ }^{60} \mathrm{Co} \gamma$-ray radiation at room temperature up to a dose of $5 \cdot 10^{7}$ Gy on the parameters of laser-generated plasma ions at the surface of single crystalline silicon and multicomponent silicate glass targets is investigated using time-of-flight mass spectrometer based on an electrostatic energy analyzer. The main impact of the radiation is observed in the charge state of the plasma ions: for small energy ions the charge increases with increasing the radiation dose, whereas the increase in the radiation dose results in the decrease of the charge of high-energy ions. The maximum energy of the ions also decreases with increasing the radiation dose. Effect of point defects created by metal atom inclusions on the absorption properties of silicate glasses is also studied. The defects decrease the resistance of the glasses to optical damage as they become centers of intense light absorption.
\end{abstract}

Keywords Laser-induced Plasma, Multi-charge Ions, Mass-spectrometer

\section{Introduction}

Defect formation in wide band gap insulators (such as amorphous $\mathrm{SiO}_{2}$ ) exposed to energetic photons (x-rays, $\gamma$-rays, intense ultraviolet radiation) has been a study object of many theoretical and experimental research works in the past (see Refs. [1, 2] for review). Such an interest is motivated by large utilization of silica-based materials for electronic and optical applications in natural and artificial radiative environments. The radiation results in a complex degradation of the properties of the materials creating, e.g., point defects. The most important radiation-induced defects in glassy silica (amorphous $\mathrm{SiO}_{2}$ ) are the $E$ ' center consisting of threefold coordinated silicon atom with an unpaired electron and the nonbridging oxygen hole center (i.e., a dangling oxygen bond) [3-6]. Despite intense research in this field, many aspects of the underlying physical processes remain largely unexplored [2]. For example, there are still open questions regarding the mechanisms for the creation of E'-centers depending on the radiation dose. Several theoretical models have been proposed, like the activation of additional precursors resulting in an exponential growth of defects [7]; the activation of the unperturbed matrix that can give rise to growth of defects without saturation [8,9]; and finally, a creation of the E'-centers with concurrent conversion to other defects to recover the initial structure. These processes have been associated with sub-linear dependence of the defect concentration on the irradiation dose [10]. Such non-linear dependence with no saturation up to higher doses has also been reported in recent electron paramagnetic resonance spectroscopy measurements [11], which are accepted to be one of the most useful tools to identify and characterize the irradiation-created paramagnetic defects. A different level of complexity is added to the context in the presence of substitutional doping [1]. For example, the metallic inclusions create extra electron-trapping centers in addition to the mechanisms mentioned above. Ge-. P-, B- and Al-doped silica glasses have been studied in the past for fiber optics applications [1, 12].

Due to its optical homogeneity and excellent transparence for high-energy photons, fused silica is extensively used for laser applications in inertial confinement fusion (ICF) programs and other high-power laser systems, such as the megajoule class fusion lasers. Defect formation in these materials is a critical issue, which can occur under different experimental conditions [13-14]. In ICF applications, the neutrons and gamma radiation produced by deuterium-tritium fusion reactions are the main sources for the silicate damage. Such preexisting defects can result in further damage during laser applications. Therefore, 
fundamental understanding the effect of defects on the light absorption and fluorescence becomes important factor for controlling the evolution of laser-induced damage and designing an effective damage resistance procedure in laser applications [15]. In particular, studying the effect of neutron and gamma radiation on the properties of silicate materials is crucial for constructing window materials for transmission lines in plasma heating and diagnostic systems in nuclear fusion reactors [16-18]. In this work, we use electrostatic mass-spectrometer to study the laser-induced damage on silica targets prior subjected to ${ }^{60} \mathrm{Co} \gamma$-ray radiation of different dose. Experiments are conducted at laser input intensities above the damage threshold, so that the effect of $\gamma$-radiation-induced defects is reflects on the parameters of laser-generated plasma particles. Radiation-induced defects affect both the charge state and energy of the plasma ions. We also consider silica glasses containing $\mathrm{Fe}$ inclusions and rare earth elements, such as $\mathrm{Ce}$ and $\mathrm{Nd}$. Non-controllable Fe inclusions were present in the system during the fabrication processes, whereas $\mathrm{Ce}$ and $\mathrm{Nd}$ elements were included in the samples using sol-gel route. Such rare earth elements, embedded into crystalline or amorphous host matrices, find wide applications in the field of optical communication technology and laser industry due to their efficient emission characteristics [19]. Silica glasses are preferred as compared to other glassy materials as it offers several additional features such as low thermal expansion, high softening, high ultraviolet transparency, strong thermal resistance, low refractive index and high surface damage threshold [20]. Our experimental results show that the resistance of silica glasses to laser damage increases with increasing the concentration of $\mathrm{Nd}$ inclusions. In addition, the effect of $\gamma$-radiation on the threshold intensity of the laser also becomes less pronounced for the higher concentration of the inclusions.

\section{Experimental Method and Sample Preparation}

Experiments were conducted using electrostatic mas-spectrometer, schematics of which are shown in Fig. 1. Targets are placed in a vacuum chamber with vacuum level not smaller than $\sim 10^{-6}$ Torr to avoid the effect of adsorbed gas atoms, water molecules and dust at the surfaces of the targets. We first cleaned the surface of the sample using the laser radiation with small intensity. The radiation from the $\mathrm{Nd}^{3+}$ glass laser with wavelength $1060 \mathrm{~nm}$ and pulse duration $50 \mathrm{~nm}$ has been focused on the surface of the target within the area of $250 \mu \mathrm{m}$ in diameter. To avoid the effect of accumulation, the interaction place of laser radiation with the target was changed after the formation of laser-produced plasma. The intensity of the neodymium glass laser, working in single shoot mode, was calibrated in the interval of $\mathrm{q}=10^{8}-10^{11} \mathrm{~W} / \mathrm{cm}^{2}$ using optical filters, which exceeds the threshold for the formation of laser-produced plasma $\left(\sim 10^{8}\right.$ $\mathrm{W} / \mathrm{cm}^{2}$ ). It is known that the damage threshold of the optical dielectrics is above $10^{9} \mathrm{~W} / \mathrm{cm}^{2}$. Therefore, in our experiments the optical damage of the targets is determined by the appearance of ions' signal in the mass-spectra. In additional, optical microscopy is used to monitor the surface morphology of the targets after the laser interaction. All measurements were performed at room temperature.

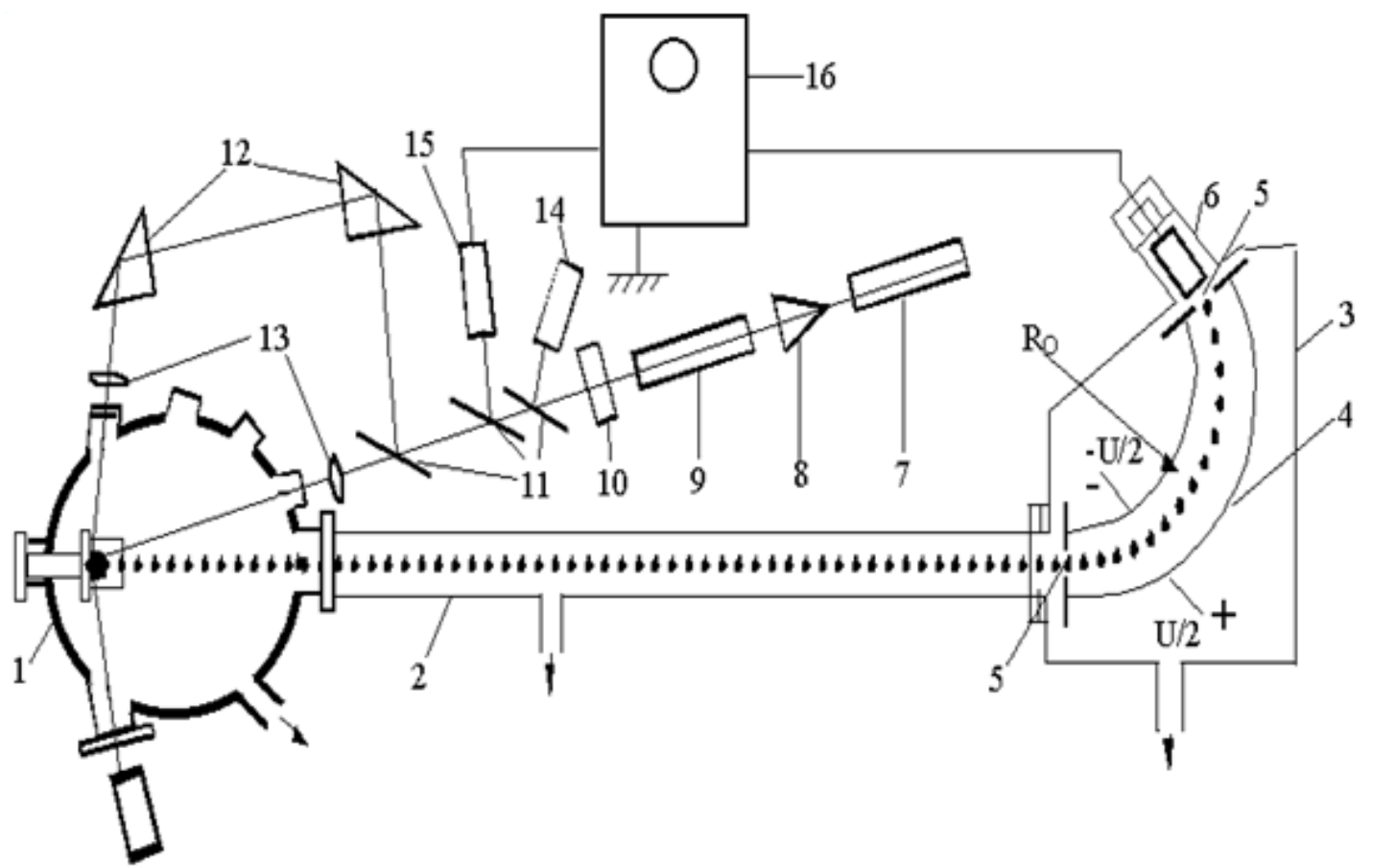

Figure 1. Schematics of the experimental setup. 1 - multifunctional target chamber, 2 - time-of-flight mass-analyzer, 3 - chamber of electrostatic analyzer, 4 - electrostatic analyzer, 5 - input and output slits, 6 - detectors, 7 - He-Ne laser, 8 - modulator, 9 - Nd:YAG laser, 10 - optical filters, 11 - parallel plates, 12 - reflectors, 13 - focusing lenses, 14 - calorimeter, 15 - coaxial photoelement, 16 - detectors. 
Three different silica grades studied in this work have commercial origin (http://www.lomo.ru): i) high purity synthetic silica, which is used as a reference in our study; ii) "naturally fused silica", obtained by fusion of natural quartz crystal containing different metallic impurities and iii) silica prepared from an acid-catalysed sol-gel solution followed by heat treatment. In the latter case $\mathrm{Ce}$ and $\mathrm{Nd}$ dopants were included in a controlled way. All the targets were fabricated in the form of a disk with thickness $5 \mathrm{~mm}$ and diameter 20 $\mathrm{mm}$. Irradiation by $\gamma$-rays was carried out at room temperature in a ${ }^{60} \mathrm{Co}$ source at a rate of $2 \mathrm{kGy} / \mathrm{h}$. Doses from $10^{4}$ Gy up to $5 \cdot 10^{7}$ Gy have been progressively accumulated in a given sample.

\section{Experimental Results for Single Crystalline Silicon Target}

In this section we study the effect of $\gamma$-radiation induced point defects on the parameters laser-induced plasma ions at the surface of metal-inclusion free samples. The reason for using single crystalline targets is to neglect to effect of metallic inclusions in the case of fused silica. In the latter case the parameters of plasma ions are strongly affected by to the presence of different elements due to their mutual interactions [21].

Figures 2 (a-e) show the mass-charge spectra of a plasma ions created on the surface of non-radiated silicon target under the laser radiation with intensity $\mathrm{q}=6 \cdot 10^{9} \mathrm{~W} / \mathrm{cm}^{2}$ for different energy (E) to charge $(\mathrm{Z})$ ratio of the ions. It is seen from these plots that the silicon ions with maximal charge of 5 can be detected at a distance of $360 \mathrm{~cm}$, which the time-of-flight distance of the setup. With increasing the E/Z ratio, the number of peaks in the spectra decreases and for larger ratio of $\mathrm{E} / \mathrm{Z}$ only single charged ions can be observed.

Figures $2(\mathrm{f}-\mathrm{h})$ present the results obtained for the samples exposed to $\gamma$-radiation of different dose. Several new features are observed in the charge spectra of the plasma ions due to the $\gamma$-radiation. For example, the maximum charge of plasma ions increases from $5^{+}$to $7^{+}$for the dose of radiation $10^{4} \mathrm{~Gy}$ (Fig. $2(\mathrm{~g})$ ). The maximum charge increases further with increasing the radiation dose (Fig. 2 (h)). Similar results have been obtained for the other energies of the ions and for different laser intensity. However, such a tendency of increasing charge with radiation dose is observed only for ions of energy $E / Z \leq 500 \mathrm{eV}$. For high energy ions $(E / Z>500$ $\mathrm{eV}$ ) the charge of the ions decreases with increasing the $\gamma$ -radiation dose. This is shown in Fig. 3, where we plot the dependence of the ions charge on the energy of the ions for different values of the radiation dose. For the non-radiated sample and also for the sample with slammer radiation dose non-monotonic dependence of the ions' charge on the energy of the ions is observed (black-square and red-circular symbols). The peak in the $Z(E)$ curve is found near $E=0.5$ $\mathrm{keV}$. At larger value of the radiation doses, the charge of the ions decreases almost linearly with increasing the energy (blue triangles). These results also show that the energy of ions of the same charge state decreases with increasing the irradiation dose, i.e., the energy spectra of ions shift to smaller energies. This suggests that in order to have ions with certain charge one should spend less energy in the case of irradiated sample as compared to non-irradiated sample, i.e. less energy of the laser radiation is necessary to obtain the ions of given charge. This also opens the possibility of increasing the density of plasma by $\gamma$-irradiation.

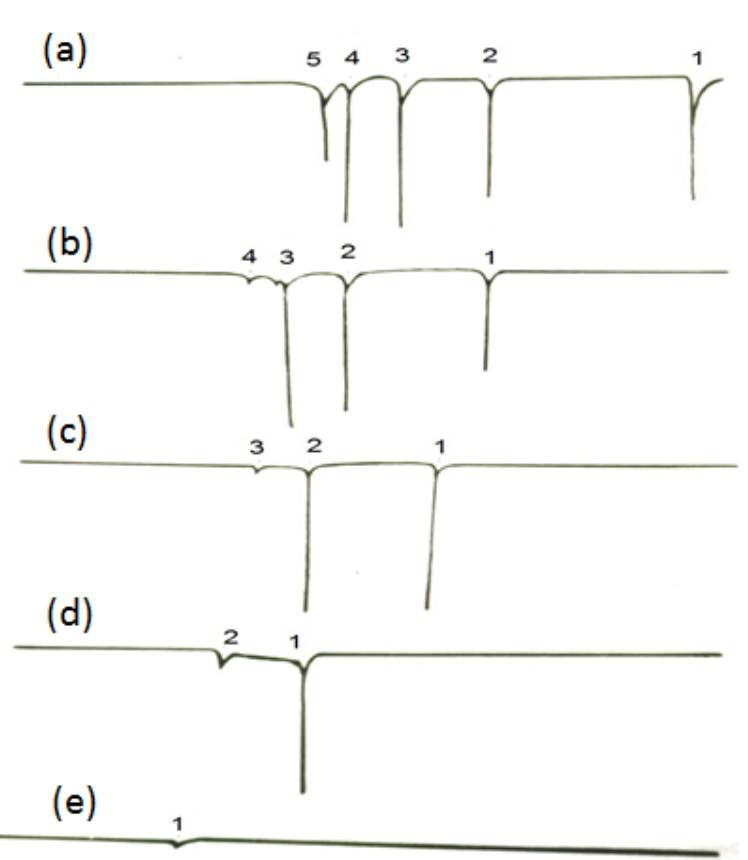

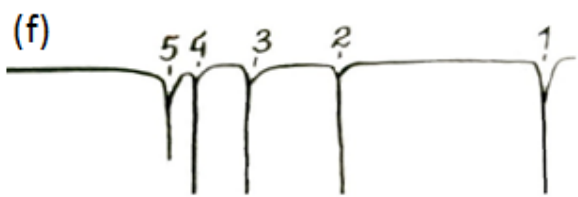

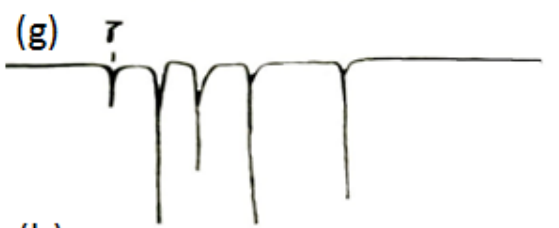

(h)

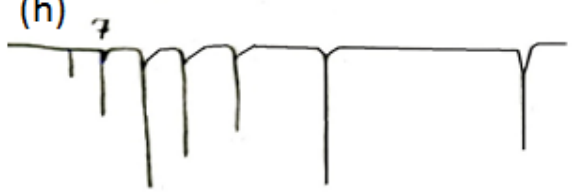

Figure 2. (a-e) Mass-charge spectra of laser-generated plasma ions from the silicon target for different energy-to-charge ratios: $\mathrm{E} / \mathrm{Z}=200 \mathrm{eV}$ (a), $400 \mathrm{eV}$ (b), $500 \mathrm{eV}$ (c), $900 \mathrm{eV}$ (d) and $2000 \mathrm{eV}$ (e). (f-g) Spectra of silicon ions with $\mathrm{E} / \mathrm{Z}=200 \mathrm{eV}$ without of $\gamma$-radiation (f) and with $\gamma$-radiation of dose $10^{4} \mathrm{~Gy}$ (g) and $10^{7} \mathrm{~Gy}(\mathrm{~h})$. All the results are obtained for $\mathrm{q}=6 \cdot 10^{9} \mathrm{~W} / \mathrm{cm}^{2}$. Numbers show the charge state of the ions. 


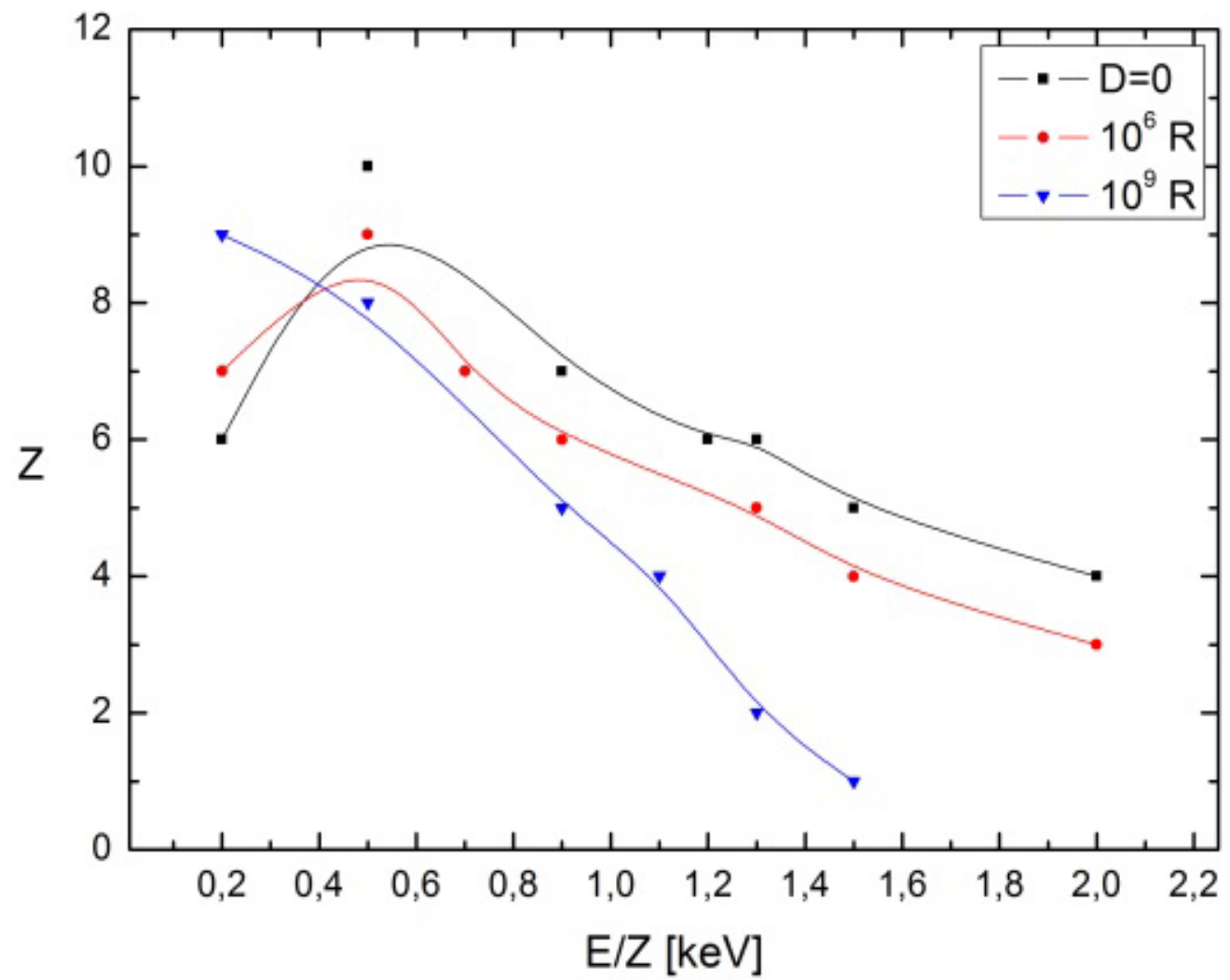

Figure 3. Energy dependence of the charge of ions in plasma created under the action of laser radiation with intensity q=5.10 $\mathrm{W} / \mathrm{cm}^{2}$ on the surface of non-radiated and $\gamma$-irradiated silicon targets with dose $10^{6} \mathrm{R}$ and $10^{9} \mathrm{R}$.
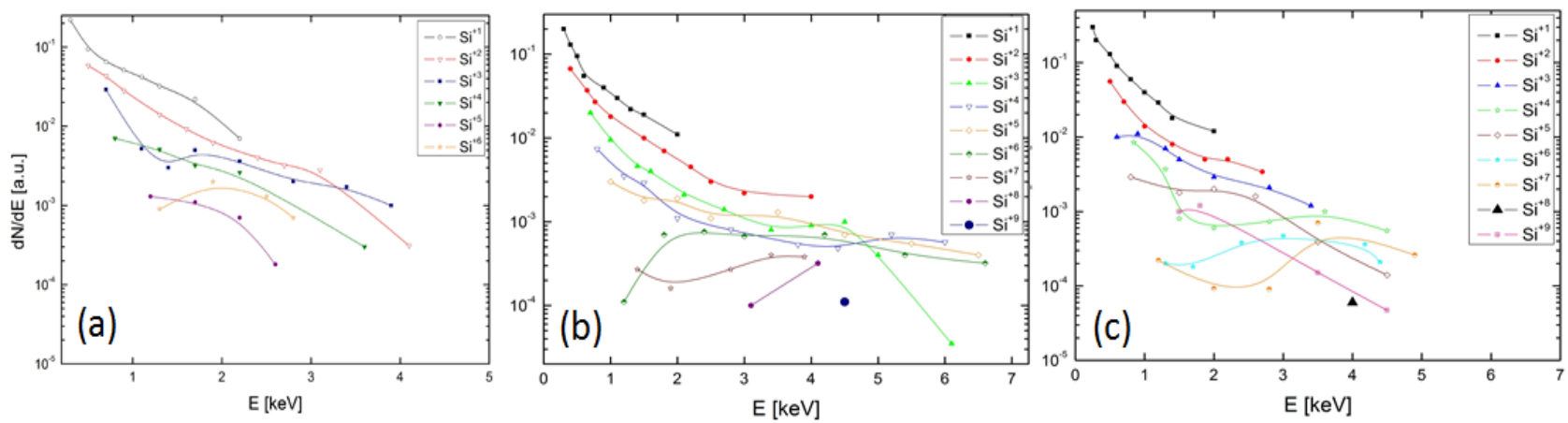

Figure 4. Energy spectra of plasma ions created under the laser radiation of intensity $\mathrm{q}=5 \cdot 10^{10} \mathrm{~W} / \mathrm{cm}^{2}$ on the surface of non-radiated (a) and $\gamma$-irradiated silicon target with doses $10^{6} \mathrm{R}(\mathrm{b})$ and $10^{9} \mathrm{R}(\mathrm{c})$.

On the base of mass-charge spectra we have constructed kinetic energy spectra of ions. Figure 4 (a) shows the energy spectra of silicon ions created on the surface of non-radiated silicon target under the action of laser radiation with intensity $\mathrm{q}=5 \cdot 10^{10} \mathrm{~W} / \mathrm{cm}^{2}$. For this value of the laser intensity, the maximum charge of the ions is 6 . Figures $4(b, c)$ show the energy spectra of ions from $\gamma$-radiated samples. It is seen from these figures that the energy spectra of ions are considerably affected by $\gamma$-radiation: both the energy range and maximum energy of the ions decreases considerably by increasing the dose of the radiation. However, the maximum energy of the ions in the latter sample is still larger than the energy of the ions in non-radiated system. Similar effect of the $\gamma$-radiation has been observed for the other intensities of the laser radiation and for all the charge multiplicities.

\section{Effect of Nd Inclusions of the Properties of Silicate Glasses}

In this section we study the effect of laser radiation on the silicate glasses prior irradiated by $\gamma$-radiation. We first analyzed the surface of the considered targets after action of the laser radiation. We found that the damage of the surface by the laser radiation occurs in the form of craters with molten edges, inside of which there are small cavities. The reason for such changes is the presence of impurity inclusions and optical inhomogeneities, which result in effective adsorption of laser energy [22]. For our glassy silica sample, the diameter of the crater was $\sim 50 \mu \mathrm{m}$. The size of the crater increases with further increasing the intensity of the laser, and reaches $\sim 300 \mu \mathrm{m}$ for $\mathrm{q}=100$ 
$\mathrm{GW} / \mathrm{cm}^{2}$, as was reported in previous experiments [23].

Depending on the intensity of the laser radiation, we have observed additional signals in the experimental mass-charge spectra corresponding to different elements of the periodic table (see Fig. 5). For example, hydrogen and oxygen signals are observed in most of the cases, indicating to the presence of water and hydroxyl groups in the studied samples [24]. The other elements (e.g. carbon and iron) are also detected in the mas-charge spectra of the ions. Since these elements are included into the targets in uncontrollable way, we included $\mathrm{Nd}$ and $\mathrm{Ce}$ ions artificially into the silicate targets and studied the effect of such inclusions on the obtained results.
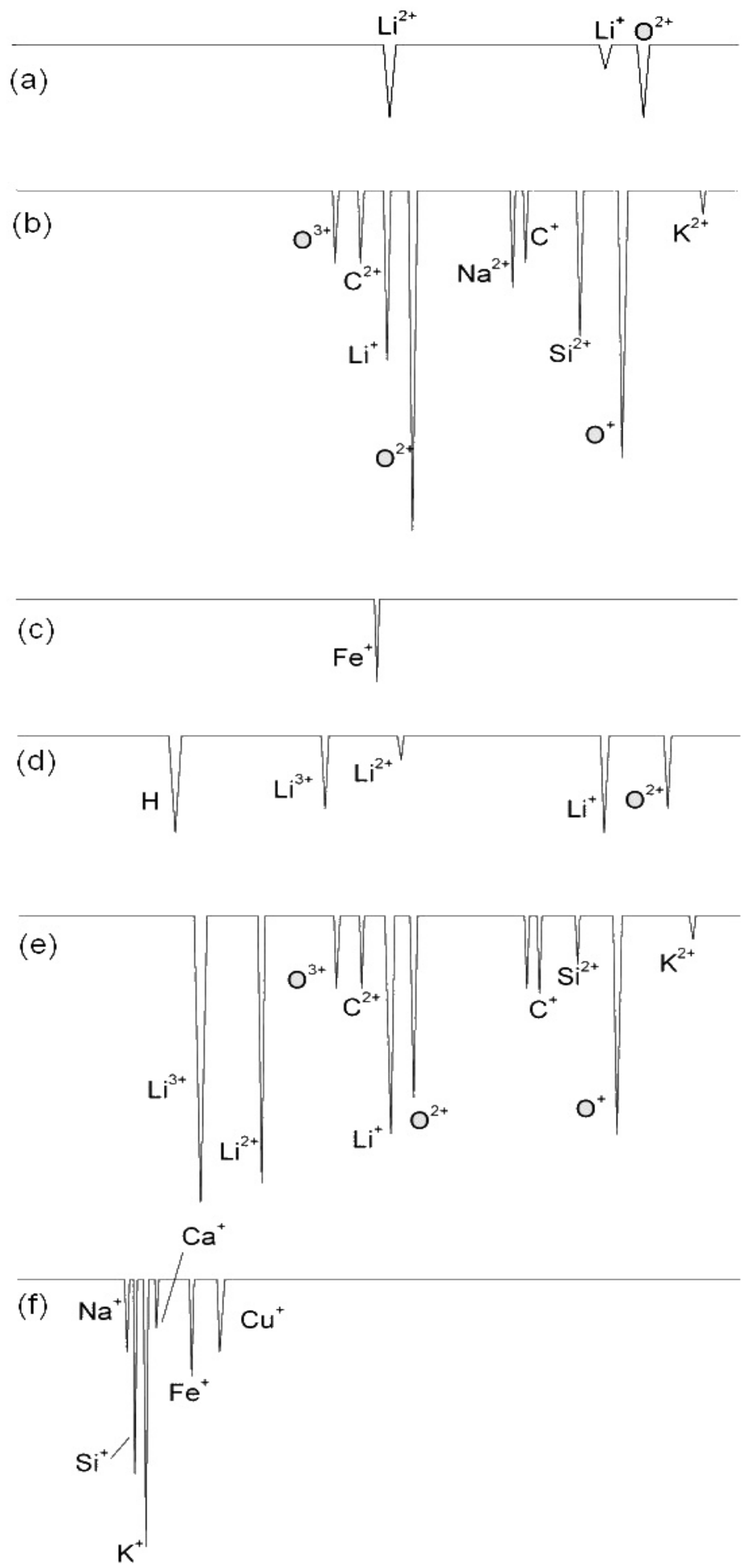

Figure 5. Mass-spectra of plasma ions, generated on the surface of silicate glass during the interaction of laser radiation with intensities $q=7 \cdot 10^{9}$ (a-b) and $9 \cdot 10^{10} \mathrm{~W} / \mathrm{cm}^{2}$ (c-f) for the energy of ions $\mathrm{E} / \mathrm{z}=50 \mathrm{eV}(\mathrm{a}-\mathrm{d}), 100 \mathrm{eV}(\mathrm{b}-\mathrm{e}), 800 \mathrm{eV}$ ( b ) and $2000 \mathrm{eV}$ ( f ). 


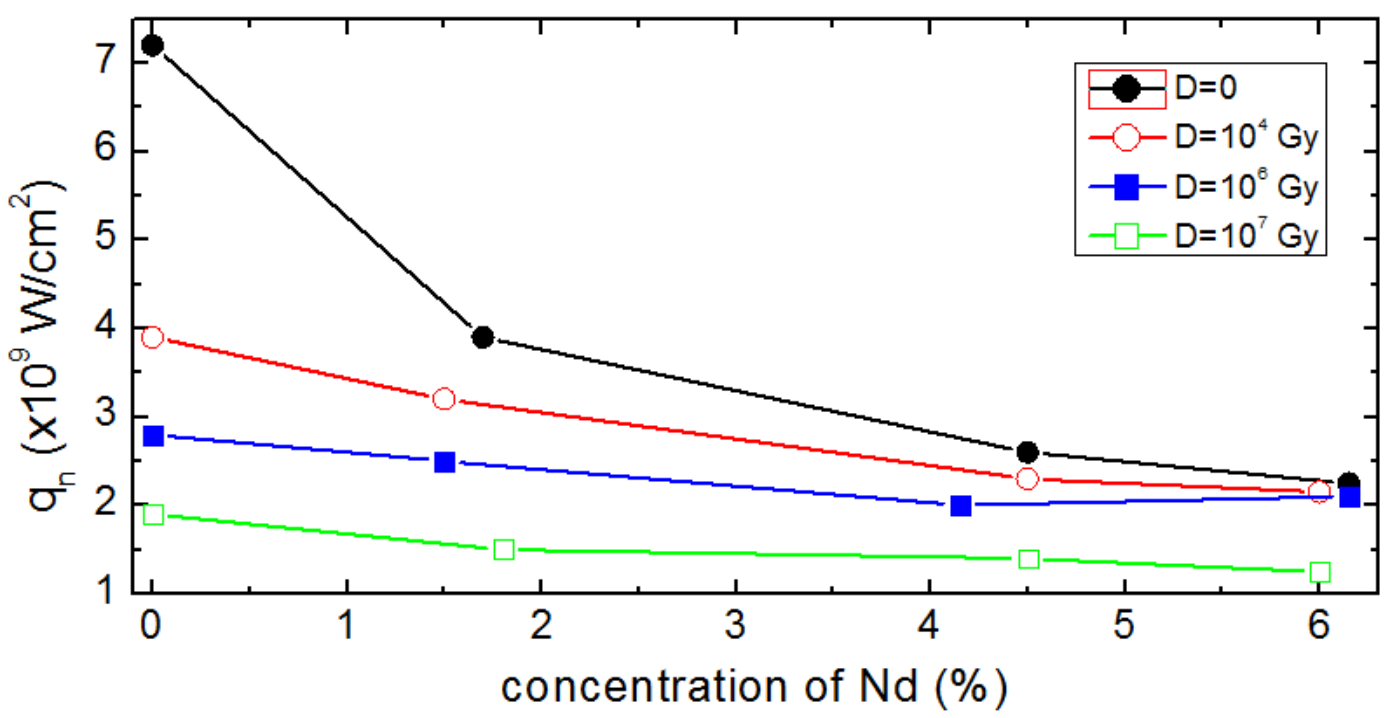

Figure 6. The critical intensity of the laser radiation corresponding for the surface destruction of the silica sample with $\mathrm{Nd}$ inclusions as a function of Nd concentration. The results are shown for different values of the gamma-radiation dose. Solid lines are the guide to the eye.

To study the effect of Nd doping on resistance of silicate glasses to laser radiation, we determined the critical intensity of the laser, $\mathrm{q}_{\mathrm{n}}$, corresponding to the destruction of the target surface. The results are shown in Fig. 6, where we plot $\mathrm{q}_{\mathrm{n}}$ as a function of the $\mathrm{Nd}$ concentration. $\mathrm{q}_{\mathrm{n}}$ is determined as a laser intensity when first ion signals are detected. It is seen from this figure that $\mathrm{q}_{\mathrm{n}}$ decreases monotonically with increasing the concentration of $\mathrm{Nd}$ ions. For example, $\mathrm{q}_{\mathrm{n}}$ drops from $7.2 \cdot 10^{9} \mathrm{~W} / \mathrm{cm}^{2}$ to $2.1 \cdot 10^{9} \mathrm{~W} / \mathrm{cm}^{2}$ with including $6 \%$ of $\mathrm{Nd}$. To our understanding, this reduction of $\mathrm{q}_{\mathrm{n}}$ is due to optical inhomogeneities created due to the fluctuations in the glass density, as well as due to the appearance of additional optical bands connected with the adsorption of spectra $\mathrm{Nd}^{3+}$ ions. The $\gamma$-radiation results in the following changes in the obtained results: i) optical adsorption spectrum and the threshold value of the laser radiation are not affected for radiation of dose $\mathrm{D} \leq 5 \cdot 10^{2}$ Gy and ii) with increasing $\mathrm{D}>$ $5 \cdot 10^{2} \mathrm{~Gy}$, the color of the glasses changes and its optical resistance decreases. The intensity of these processes depends of the radiation dose. For example, with increasing the dose of the radiation from $5 \cdot 10^{2}$ Gy to $10^{7} \mathrm{~Gy}, \mathrm{q}_{\mathrm{n}}$ decreases by an order of magnitude.

To compare with the results obtained for $\mathrm{Nd}$ doped samples, we also studied the samples with Ce inclusions. Experimental results show that the resistance of the $\mathrm{Ce}$ doped samples to laser radiation increases as compared to $\mathrm{Nd}$ doped samples. For example, the size of the cracks at the surface of the sample becomes considerably smaller and the threshold laser intensity $\mathrm{q}_{\mathrm{n}}$ increases by at least $10 \%$. Similar results are obtained for all considered doses of the $\gamma$-radiation.

However, non-controllable metallic inclusions, e.g., Fe inclusions, considerably reduce the optical resistance of the samples as compared to $\mathrm{Nd}$ and $\mathrm{Ce}$ doped samples. The size of the craters and cavities also become larger in the former case. The effect of $\gamma$-radiation also becomes more pronounced in samples with $\mathrm{Fe}$ inclusions. For example, gamma radiation results in strong coloring of the samples, increased background adsorption in the range of 850-1200 $\mathrm{nm}$ and appearance of additional intense gap in the absorption with a maximum at $1100 \mathrm{~nm}$. With increases the dose of the irradiation above $\mathrm{D}>10^{6} \mathrm{~Gy}$, additional gap appeared in the optical spectra with maximum at $215 \mathrm{~nm}$. Its intensity increases with increasing the radiation dose.

Analysis of the data about the effect of gamma irradiation on the critical laser intensity $\mathrm{q}_{\mathrm{n}}$ shows one characteristical feature for both clean and doped samples: monotonic decrease of $\mathrm{q}_{\mathrm{n}}$ and increase of the size of the craters occur only for doses $\mathrm{D}=10^{8} \mathrm{R}$. At higher doses the intensity of surface destruction and degradation of $\mathrm{q}_{\mathrm{n}}$ further increases. The destruction is correlated with the appearance of additional adsorption gaps in the optical spectrum of gamma-irradiated samples with maximum at $215 \mathrm{~nm}$.

\section{Discussions}

To our knowledge, the negative role of $\mathrm{Fe}$ ions on the resistance and optical absorption of $\gamma$-radiated glasses can be explained as follows. It is known that $\mathrm{Fe}$ can exists in glasses in different forms. The appearance of additional gap in the adsorption spectra of the sample with maximum at $1100 \mathrm{~nm}$ and increase of its intensity with increasing the radiation indicates to the fact that gamma radiation results in inovalent transition of $\mathrm{Fe}$ ions. For example, it is possible that $\mathrm{Fe}^{3+}$ ions capture additional electron during the irradiation and forms $\mathrm{Fe}^{2+}[25,26]$, which adsorbs the radiation at $1100 \mathrm{~nm}$. As the wavelength of the laser radiation is $1060 \mathrm{~nm}$, the formation of defects in the sample which adsorbs this radiation, should lead to increase of efficiency of the degradation process of light resistance of $\gamma$-radiated glasses.

Experimental results show that the degradation process of optical resistance of $\gamma$-irradiated glasses can be slowed down by including $\mathrm{Ce}$ ions. The analysis of optical adsorption 
spectra of non Ce activated sample show that $\gamma$-radiation results in formation of defects which absorb the electromagnetic radiation in ultraviolet, optical and infrared range of the spectra. The high temperature plasma, generated during the interaction of laser radiation is an effective source of optical radiation covering all the optical part of the spectra. The formation of defects in $\gamma$-radiated samples result in addition heating of the sample and promotes initial destruction. Introducing of $\mathrm{Ce}$ during the gamma radiation affects the electron capturing process of the other defects. This results in decrease of the defect formation process, which can absorb the laser radiation in addition to plasma radiations. To our knowledge, this can explain the experimental fact that the presence of $\mathrm{Ce}$ ions results in reduction of the surface destruction.

We also found that independently on the type and concentration of the inclusions, there is a change in the dependence of $\mathrm{q}_{\mathrm{n}}$ on the dose of radiation for $\mathrm{D} \geq 3 \cdot 10^{6} \mathrm{~Gy}$. This change is correlated with the formation of additional gaps in the optical spectra of $\gamma$-irradiated sample with maximum of $215 \mathrm{~nm}$. This is connected by the formation of additional centers which adsorbs in this range (i.e., E-centers) $[27,28]$. The formation of such defects in the glass indicates to the formation of structural defects for the doses above $\mathrm{D} \geq$ $3 \cdot 10^{6} \mathrm{~Gy}$. The intensity of this process, which is connected with the bond breaking between silicon and oxygen atoms and the distortion of the first coordination sphere of the ion-glass former [28], increases with increasing the radiation dose. As a result of structural defect formation process and formation of oxygen vacancies, uniform distribution of the potential energy of the glass matrix changes [22]. Coulomb forces distort the bonds between silicon and oxygen. The tension formed in this process leads to the increase of the degradation process of sample surface strength. The increase of the concentration of E-centers, which take place at doses $\mathrm{D} \geq 3 \cdot 10^{6} \mathrm{~Gy}$, results in sharp drop in $\mathrm{q}_{\mathrm{n}}$ and dramatic destruction of the target surface.

\section{Conclusions}

Mass-spectrometric method is used to study the effect of gamma radiation, as well as the effect of metallic inclusions on the absorption properties and light resistance of silicate glasses. Depending on the dose of the $\gamma$-radiation, the surface resistance of the samples to the laser radiation decreases considerably due to the surface erosion or crack formation. Different metallic inclusions $(\mathrm{Nd}, \mathrm{Fe}$ and $\mathrm{Ce}$ ) further decreases the resistance of the glasses as these inclusions become centers of intense light absorption.

\section{REFERENCES}

[1] D. L. Griscom, J. Non Cryst. Solids 357, 1945 (2011).

[2] S. Girard, J. Kuhnhenn, A. Gusarov, B. Brichard, M. Van Uffelen, Y. Ouerdane, A. Boukenter, and C. Marcandella,
IEEE TRANSACTIONS ON NUCLEAR SCIENCE 60, 3 (2013).

[3] R. A. Weeks, J. Appl. Phys. 27, 1376 (1956)

[4] E. J. Friebele, D. L. Griscom, M. Stapelbroek, and R. A. Weeks, Phys. Rev. Lett. 42, 1346 (1979).

[5] D. L. Griscom, J. Non-Cryst. Solids 149, 137 (1992).

[6] H. H. Mahmud, A. Mansour, and F. M. Ezz-Eldin, J. Radioanal Nucl. Chem. 302, 261 (2014).

[7] D. L. Griscom, M. E. Gingerish, and E. J. Friebele Phys. Rev. Lett. 71, 1019 (1993).

[8] H. Imai and H. Hirashima J. Non Cryst. Solids 179, 202 (1994).

[9] F. L. Galeener, D. B. Kerwin, A. J. Miller and J.C. Mikkelsen Jr. Phys. Rev. B 47, 7760 (1993).

[10] V. A. Mashkov, W. R. Austin, L. Zhang, and R. G. Leisure, Phys. Rev. Lett. 76, 2926 (1996).

[11] S. Agnello, N. Chiodin, A. Paleari, and A. Parlato, J. Non Cryst. Solids 353, 573 (2007).

[12] J. C. Lagomacini, D. Bravo, A. Martín, F. J. López, P. Martín, and A. Ibarra, J. Non-Cryst. Solids 403, 5 (2014).

[13] S. T. Yang et al., Applied Optics 49, 2606 (2010).

[14] T. Shikama and S. J. Zinkle, Journal of Nuclear Materials 307, 1073 (2002).

[15] C. B. Norris and E. P. Eernisse, J. Appl. Phys. 45, 3876 (1974).

[16] L. Douillard and J.P. Durand, J. Phys. III France 6, 1677 (1996).

[17] H. Sugiura et al., J. Appl. Phys. 81, 1651 (1997).

[18] R. Devanathan, Nuclear Instruments and Methods in Physics Research B, 267, 3017(2009).

[19] R. Reisfeld, C.K. Jorjensen, Laser and Excited States of Rare Earth, Springer, New York, 1977.

[20] S. S. Wang, Y. Zhou, Y. L. Lam, C. H. Kam, Y. C. Chan, and X. Yao, Mater. Res. Innov. 1, 92 (1997).

[21] R.T. Khaydarov, H.B. Beisinbaeva, M.M. Sabitov, V.B. Terentev, F.M. Tojinazarov, Nucl. Fusion 49, 065022 (2009).

[22] A. Kh. Islamov, E. M. Ibragomova and I. Nuritdinov. Journal of Nuclear materials 363, 1023 (2007).

[23] H. B. Beisinbaeva, M. M. Sabitov, and R. T. Khaydarov, Proceedings of "The Third Eurasian Conference "Nuclear science and its application", October 5-8, 2004, Tashkent, Uzbekistan.

[24] B. Brichard, A. L. Tomashuk, V. A. Bogatyrjov et al., Journal of Non-Crystaline Solids 353, 466 (2007).

[25] T. T. Turdiev, J. D. Ibragimov, and I. Nuritdinov, Uzbek journal of physics 7, 105 (2005).

[26] I. M. Burjinskii, L. I. Avakyants and V. F. Surkova, Russian journal of applied spectroscopy 22, 248 (1975).

[27] F. J. Feigl, D. Fowler, and K. H. Yip, Sol. Stat. Comm. 14, 225 (1974).

[28] S. M. Brekhovskikh, V. A. Tyul'nin, E. K. Mamedov, Russian journal of physics and chemistry of glasses 4, 443 (1987). 